EUROPEAN ORGANIZATION FOR NUCLEAR RESEARCH

CERN-EP/98-127

August 17, 1998

\title{
Upper Limit on the Lifetime Difference of Short- and Long-lived $\mathrm{B}_{\mathrm{s}}^{0}$ Mesons
}

The L3 Collaboration

\begin{abstract}
An upper limit on the lifetime difference of short- and long-lived $\mathrm{B}_{\mathrm{s}}^{0}$ mesons has been obtained using an inclusive bottom hadron sample from 2 million hadronic $\mathrm{Z}$ decays collected by the L3 experiment at LEP. A lifetime fit has been performed on data samples separately enriched in neutral and charged b hadrons. An experimental upper limit on the decay rate difference of short- and long-lived $\mathrm{B}_{\mathrm{s}}^{0}$ mesons of

$$
(|\Delta \Gamma| / \Gamma)_{\mathrm{B}_{\mathrm{s}}^{0}}<0.67 \quad \text { (95\% C.L.) }
$$

has been determined. In addition, the lifetimes of $\mathrm{B}^{+}$and $\mathrm{B}_{\mathrm{d}}^{0}$ mesons have been measured to be $\tau\left(\mathrm{B}^{+}\right)=1.66 \pm 0.06 \pm 0.03 \mathrm{ps}$ and $\tau\left(\mathrm{B}_{\mathrm{d}}^{0}\right)=1.52 \pm 0.06 \pm 0.04 \mathrm{ps}$, where the first errors are statistical and the second are systematic.
\end{abstract}

Submitted to Phys. Lett. B 


\section{Introduction}

Neutral mesons such as $\mathrm{K}^{0}, \mathrm{~B}_{\mathrm{d}}^{0}$ and $\mathrm{B}_{\mathrm{s}}^{0}$ have, beside CP-violating decays, two interesting properties: the flavour eigenstates oscillate into one another with a frequency ${ }^{1)} \omega$, and the CP eigenstates decay with two distinct lifetimes $\tau_{1}, \tau_{2}$. The situation of neutral B mesons, $\mathrm{B}_{\mathrm{d}}^{0}$ and $\mathrm{B}_{\mathrm{s}}^{0}$, is qualitatively equivalent to that of the well studied neutral kaon system. However, the numerical values of the mass difference $\Delta M=\omega$ and the decay rate difference $\Delta \Gamma=1 / \tau_{1}-1 / \tau_{2}$ are rather different. The oscillation behaviour of $\mathrm{B}_{\mathrm{d}}^{0}$ mesons is well established [1-3]. The decay rate difference is expected to be much smaller as compared to the kaon system and has not yet been investigated. Thus, it is of great interest to search for two distinct lifetimes of neutral B mesons.

This letter points out that the inclusive decay time distribution of $b$ hadrons at LEP is sensitive to the decay rate difference $\Delta \Gamma$ of neutral $B$ mesons. Previous measurements of the average $\mathrm{b}$ hadron lifetime $[4,5]$ have been used to derive a value of the matrix element $\left|\mathrm{V}_{\mathrm{cb}}\right|$ of the Cabibbo-Kobayashi-Maskawa (CKM) matrix [6]. The inclusive decay time distribution at long time scales also provides information on $\Delta \Gamma$. For example at LEP, a value of $\Delta \Gamma / \Gamma=0.5$ for $\mathrm{B}_{\mathrm{s}}^{0}$ mesons would produce a $10 \%$ change in the total number of $\mathrm{b}$ events with lifetimes greater than seven average lifetimes compared to the expected number for $\Delta \Gamma / \Gamma=0$.

The inclusive $\mathrm{b}$ hadron sample at LEP consists of $\mathrm{B}_{\mathrm{d}}^{0}, \mathrm{~B}_{\mathrm{s}}^{0}$ and $\mathrm{B}^{+}$mesons and $\mathrm{b}$ baryons. Within the framework of the Standard Model, a hierarchy in the $\Delta \Gamma$ values of $\mathrm{B}_{\mathrm{d}}^{0}$ and $\mathrm{B}_{\mathrm{s}}^{0}$ mesons is expected according to: $\Delta \Gamma_{\mathrm{B}_{\mathrm{s}}^{0}} \gg \Delta \Gamma_{\mathrm{B}_{\mathrm{d}}^{0}}$. The range of theoretically expected values for $(\Delta \Gamma / \Gamma)_{\mathrm{B}_{\mathrm{s}}^{0}}$ is 0.07 to $0.27[7,8]$. The results of this letter are given in terms of $\Delta \Gamma_{\mathrm{B}_{\mathrm{s}}^{0}}$, assuming that the value of $\Delta \Gamma_{B_{d}^{0}}$ is negligible. An inclusive charge measurement has been applied to enrich different data samples in neutral and charged $b$ hadrons. The decay time distributions of these samples have been fitted by $\Delta \Gamma_{\mathrm{B}_{\mathrm{s}}^{0}}$ and the lifetimes of $\mathrm{B}^{+}$and $\mathrm{B}_{\mathrm{d}}^{0}$ mesons, with the average lifetimes of $\mathrm{B}_{\mathrm{s}}^{0}$ mesons and $\mathrm{b}$ baryons taken from previous measurements [9-11].

\section{Lifetime and Charge Measurement}

The L3 detector has been described elsewhere [12]. The selection of hadronic events was similar to the one used for the measurement of the total hadronic cross section [13]. The thrust axes of the jets used in this analysis were required to be in the polar angular range $|\cos \theta|<0.74$. The reconstruction of the $b \bar{b}$ production vertex and the $b$ decay vertices was performed with the same method applied in the measurement of the average $b$ hadron lifetime $[5,14]$. Important features of this analysis are summarised in the following. Tracks were required to have a silicon microvertex detector measurement. They were divided into three groups corresponding to the primary $\mathrm{e}^{+} \mathrm{e}^{-}$annihilation location and the two secondary $\mathrm{b}$ decay locations in a $\mathrm{Z} \rightarrow$ $\mathrm{b} \overline{\mathrm{b}}$ event. The groups were obtained using the impact parameter and the rapidity measurements of the tracks. The vertex positions were calculated by minimising the impact parameters from each group. The track impact parameter resolutions at high momentum have been measured as $32 \mu \mathrm{m}$ in the bending plane of the magnetic field, the $r \phi$ plane, and $300 \mu \mathrm{m}$ in the $r z$ plane parallel to the beam direction, the $z$ axis. The additional multiple scattering error was $110 \mu \mathrm{m} /\left(p_{\perp}(\mathrm{GeV}) \sqrt{\sin \theta}\right)$ in both projections, where $\theta$ is the polar angle of the track.

The analysis presented here was based on a data sample of $2.0 \times 10^{6}$ hadronic events from the years 1994 and 1995, corresponding to an integrated luminosity of $79.7 \mathrm{pb}^{-1}$. Out of these,

\footnotetext{
${ }^{1)}$ Throughout this paper we use $\hbar=c=1$.
} 
$1.1 \times 10^{6}$ events suitable for a lifetime measurement have been selected. The vertex locations have been reconstructed for $445 \mathrm{k}$ events that have at minimum four selected tracks per event hemisphere. Corresponding Monte Carlo events have been generated using the JETSET 7.4 program [15], and the response of the L3 detector was modelled with the GEANT detector simulation program [16].

The measurement of the b decay time is based on the impact parameters of tracks reconstructed at the secondary vertices (SV). The impact parameter is well suited for a b lifetime measurement due to its small dependence on the $\mathrm{b}$ fragmentation. It was defined here as the error-weighted average of the impact parameters of all tracks from a secondary vertex. The reference point was the primary event vertex (PV), calculated individually for each event. The impact parameter distribution was signed in the usual convention by whether the track crossed the jet axis in front of or behind the primary event vertex.

A statistical reconstruction of the secondary vertex charge $Q_{S V}$ has been used to separate the impact parameter distributions of neutral and charged b hadrons. The separation gives a higher statistical sensitivity to the $\Delta \Gamma$ value of neutral $B$ mesons and includes the possibility of a cross check by a reference measurement of the data distributions. The secondary vertex charge $Q_{S V}$ has been defined as the sum of charges $Q_{i}$ for all tracks $i=1, \cdots, N_{S V}$ associated with that vertex, weighted by the probabilities $\xi_{i}^{S V}$ that the tracks originate from it:

$$
Q_{S V}=\operatorname{sign}\left(Q_{J}\right) \sum_{i=1}^{N_{S V}} Q_{i} \xi_{i}^{S V}
$$

The probability $\xi_{i}^{S V}$ was determined from the $\chi^{2}$ vertex fit [5]:

$$
\xi_{i}^{j}=\exp \left\{-\frac{1}{2}\left[\left(\frac{\delta_{i}^{r \phi}\left(\vec{x}_{j}\right)}{\sigma_{i}^{r \phi}}\right)^{2}+\left(\frac{\delta_{i}^{z}\left(\vec{x}_{j}\right)}{\sigma_{i}^{z}}\right)^{2}\right]\right\} P_{j}\left(\eta_{i}\right) \quad \mathrm{i}=1, \cdots, \mathrm{N}_{\mathrm{SV}} \quad \mathrm{j}=\mathrm{PV}, \mathrm{SV} .
$$

The probabilities were normalised such that $\xi_{i}^{P V}+\xi_{i}^{S V}=1$ for each track. The parameter $\delta_{i}^{r \phi}$ denotes the impact parameter of the track in the $r \phi$ plane with respect to the primary or secondary vertex. The parameter $\delta_{i}^{z}$ describes the difference of the $z$ position of the track at its point of closest approach in the $r \phi$ plane with respect to the $z$ position of the vertices. The associated errors are denoted by $\sigma_{i}^{r \phi}$ and $\sigma_{i}^{z}$. The last term, $P_{j}\left(\eta_{i}\right)$, describes the probability that the measured track rapidity $\eta_{i}$ corresponds to a primary or a secondary track.

In our definition of $Q_{S V}$, the sign is measured with respect to the sign of the jet charge $Q_{J}$ :

$$
Q_{J}=\sum_{i=1}^{N_{\text {jet }}} Q_{i} p_{i \|}
$$

It is defined as the sum of charges $Q_{i}$ from all tracks $i=1, \cdots, N_{j e t}$ contained in a jet (from primary and secondary vertices), weighted by the track momentum component $p_{i \|}$ parallel to the jet axis. The weighted sum of charges $\sum Q_{i} \xi_{i}$ and $Q_{J}$ are both sensitive to the charge sign of $\mathrm{B}^{+}$and $\mathrm{B}^{-}$hadrons. The product of the signs of $\sum Q_{i} \xi_{i}$ and $Q_{J}$ thus contains information on the accuracy of the charge measurement for charged $b$ hadrons. It has been used to increase the statistical separation of neutral and charged $b$ hadrons.

The charged sample contained the vertices with $Q_{S V}>0.5$ where the signs of $\sum Q_{i} \xi_{i}$ and $Q_{J}$ agreed. The neutral sample consisted of the vertices with $-0.8<Q_{S V}<0.5$. The rest of the vertices with $Q_{S V}<-0.8$ were combined in a third category with a flavour composition similar to the average $\mathrm{b}$ hadron sample in $\mathrm{Z}$ decays. The distributions of the $Q_{S V}$ values in 
data and in Monte Carlo are shown in Fig. 1. It has been observed in the Monte Carlo that the charge measurement improved with increasing impact parameter. For impact parameters larger than $300 \mu \mathrm{m}$, about one average b lifetime, the accuracy of the charge measurement reached a constant value.

A further division has been performed to enrich these samples in $b \bar{b}$ events. Each charge sample was divided into two further categories depending upon the value of the decay length in the opposite hemisphere. The b-tagged (b anti-tagged) samples were obtained by requiring the decay length $l_{\text {opp }}$ in the opposite hemisphere to be larger (smaller) than $2 \mathrm{~mm}$. The decay length was defined here as the three-dimensional distance between the primary and secondary vertices.

\section{Lifetime Fit}

The $\Delta \Gamma_{\mathrm{B}_{\mathrm{s}}^{0}}$ value was obtained from a binned $\chi^{2}$ fit to the impact parameter distributions of the six data subsamples. The numbers of vertices contained in these samples and their respective $\mathrm{b}$ quark and $\mathrm{B}^{+}, \mathrm{B}_{\mathrm{d}}^{0}$, and $\mathrm{B}_{\mathrm{s}}^{0}$ purities are summarised in Table 1. The impact parameter distributions of all six samples in the range from -3 to $+3 \mathrm{~mm}$ have been fitted simultaneously using five B lifetime parameters. These were:

1. the $\mathrm{B}_{\mathrm{s}}^{0}$ meson decay rate difference $|\Delta \Gamma| / \Gamma$.

2. the average $\mathrm{B}^{+}$and $\mathrm{B}_{\mathrm{d}}^{0}$ meson lifetime ${ }^{2)}\left\langle\tau_{\mathrm{B}}^{\mathrm{u}, \mathrm{d}}\right\rangle=\frac{1}{2}\left(\tau\left(\mathrm{B}^{+}\right)+\tau\left(\mathrm{B}_{\mathrm{d}}^{0}\right)\right)$.

3. the lifetime ratio $r=\tau\left(\mathrm{B}^{+}\right) / \tau\left(\mathrm{B}_{\mathrm{d}}^{0}\right)$.

4. the average $\mathrm{B}_{\mathrm{s}}^{0}$ meson lifetime $\tau\left(\mathrm{B}_{\mathrm{s}}^{0}\right)$.

5. the average $\mathrm{b}$ baryon lifetime $\tau\left(\Lambda_{\mathrm{b}}\right)$.

The lifetimes of $\mathrm{B}_{\mathrm{s}}^{0}$ and $\Lambda_{\mathrm{b}}$ hadrons have been constrained to the measured values from exclusive measurements [9-11]: $\tau\left(\mathrm{B}_{\mathrm{s}}^{0}\right)=1.49 \pm 0.06$ ps and $\tau\left(\Lambda_{\mathrm{b}}\right)=1.22 \pm 0.05 \mathrm{ps}$. The average lifetime $\left\langle\tau_{\mathrm{B}}^{\mathrm{u}, \mathrm{d}}\right\rangle$ and the lifetime ratio $\tau\left(\mathrm{B}^{+}\right) / \tau\left(\mathrm{B}_{\mathrm{d}}^{0}\right)$ have been chosen rather than $\tau\left(\mathrm{B}^{+}\right)$and $\tau\left(\mathrm{B}_{\mathrm{d}}^{0}\right)$ because of smaller correlations among the fit variables in the former case.

The lifetime-dependent expected distributions have been obtained from a Monte Carlo simulation. The lifetime dependence was introduced by reweighting the entries of the Monte Carlo impact parameter distribution as a function of the $b$ decay proper time values. Non-zero values of $\Delta \Gamma$ for $\mathrm{B}_{\mathrm{s}}^{0}$ mesons have been created in the Monte Carlo by the weight:

$$
\frac{\tau_{M C}}{2}\left[\frac{1}{\tau_{1}} \frac{e^{-t / \tau_{1}}}{e^{-t / \tau_{M C}}}+\frac{1}{\tau_{2}} \frac{e^{-t / \tau_{2}}}{e^{-t / \tau_{M C}}}\right],
$$

where $\tau_{M C}$ is the $\mathrm{B}_{\mathrm{s}}^{0}$ lifetime with which the Monte Carlo was generated. The lifetimes $\tau_{1}, \tau_{2}$ are the values of the $\mathrm{CP}$-even and -odd eigenstates of the $\mathrm{B}_{\mathrm{s}}^{0}$ meson. Their values are related to the average $\mathrm{B}_{\mathrm{s}}^{0}$ lifetime $\tau\left(\mathrm{B}_{\mathrm{s}}^{0}\right)$ and the rate difference $\Delta \Gamma / \Gamma$ via $^{3)}$ :

$$
\tau_{1,2}=\tau\left(\mathrm{B}_{\mathrm{s}}^{0}\right)(1 \pm \Delta \Gamma / 2 \Gamma)
$$

\footnotetext{
${ }^{2)}$ The value $\left\langle\tau_{\mathrm{B}}^{\mathrm{u}, \mathrm{d}}\right\rangle$ is not the average lifetime of all $\mathrm{b}$ hadrons in $\mathrm{Z}$ decays which was measured in our previous publication [5] due to the presence of $\mathrm{B}_{\mathrm{s}}^{0}$ and $\mathrm{b}$ baryons in the latter.

${ }^{3)}$ There is an ambiguity in the interpretation of the measured average $\mathrm{B}_{\mathrm{s}}^{0}$ lifetime $\tau\left(\mathrm{B}_{\mathrm{s}}^{0}\right)$. In the previous measurements of $\tau\left(\mathrm{B}_{\mathrm{s}}^{0}\right)$ [9] it has been assumed that $\Delta \Gamma_{\mathrm{B}_{\mathrm{s}}^{0}}=0$. Due to this assumption the actual value of $\tau\left(\mathrm{B}_{\mathrm{s}}^{0}\right)$ may differ from the measured one depending on the $\Delta \Gamma_{\mathrm{B}_{\mathrm{s}}^{0}}$ value. The influence of this effect on the fit result is discussed below.
} 
The weight (Equ. 4) is invariant under the transformation $\Delta \Gamma \rightarrow-\Delta \Gamma$. The sign of $\Delta \Gamma$ cannot be measured since the $\mathrm{CP}$ eigenstates of the $\mathrm{B}_{\mathrm{s}}^{0}$ meson are not distinguished in our method. Hence we used $|\Delta \Gamma| / \Gamma$ as fit parameter.

In the description of the average $\mathrm{b}$ hadron composition in the total $\mathrm{Z} \rightarrow \mathrm{b} \overline{\mathrm{b}}$ sample we followed the suggestions of the Particle Data Group [17]. The b hadron sample was described by the fraction $f_{\mathrm{B}_{\mathrm{s}}^{0}}$ of $\mathrm{B}_{\mathrm{s}}^{0}$ mesons, the fraction $f_{\Lambda_{\mathrm{b}}}$ of $\mathrm{b}$ baryons, and assumes equal production of $\mathrm{B}^{+}$and $\mathrm{B}_{\mathrm{d}}^{0}$ mesons, $f_{\mathrm{B}^{+}}=f_{\mathrm{B}_{\mathrm{d}}^{0}}=\left(1-f_{\mathrm{B}_{\mathrm{s}}^{0}}-f_{\Lambda_{\mathrm{b}}}\right) / 2$. The values of $f_{\mathrm{B}_{\mathrm{s}}^{0}}=0.105_{-0.017}^{+0.018}$ and $f_{\Lambda_{\mathrm{b}}}=0.101_{-0.031}^{+0.039}$ have been used as constraints in the fit.

Twelve other parameters have been simultaneously determined. These were the values $f_{b, 1}, f_{b, 2}, f_{b, 3}$ of the $\mathrm{b}$ purities in the three b-enriched samples, eight resolution correction parameters and the average number $\left\langle n_{K}\right\rangle$ of $\mathrm{K}_{\mathrm{S}}^{0}$ and $\Lambda$ decays per hadronic $\mathrm{Z}$ event. The fit parameters $f_{b, 1}, f_{b, 2}, f_{b, 3}$ agree with the Monte Carlo simulation as shown in Table 1 . The Monte Carlo impact parameter resolution was convoluted with two additional Gaussian functions for $\mathrm{b}$ and non-b events. The eight parameters of these Gaussian functions and the $\mathrm{K}_{\mathrm{S}}^{0} / \Lambda$ production rate $\left\langle n_{K}\right\rangle$ have been determined in a similar way as in our previous publication [5] and the results agree.

The fit results for the lifetime parameters are:

$$
\begin{gathered}
|\Delta \Gamma| / \Gamma=0.00_{-0.00}^{+0.30} \\
\left\langle\tau_{\mathrm{B}}^{\mathrm{u}, \mathrm{d}}\right\rangle=1.59 \pm 0.02 \mathrm{ps}, \quad \tau\left(\mathrm{B}^{+}\right) / \tau\left(\mathrm{B}_{\mathrm{d}}^{0}\right)=1.09 \pm 0.07 .
\end{gathered}
$$

The quoted errors are statistical only. A graphical representation of the fit is shown in Fig. 2. The data impact parameter distributions are compared with the Monte Carlo distribution for the fitted parameter values in four subsamples, the charged and neutral B samples and the $\mathrm{b}$ tagged and b anti-tagged samples. Good agreement is observed in all distributions over the entire impact parameter range. The $\chi^{2}$ value of the fit, normalised to the number of degrees of freedom, was 0.95. Fig. 3 shows the ratio of the data over the fit result for positive impact parameters in the b-tagged, neutral B enriched subsample. The data are consistent with a single exponential decay distribution for $\mathrm{B}_{\mathrm{s}}^{0}$ mesons.

The statistical error on $|\Delta \Gamma| / \Gamma$ was derived as follows. A probability density function (p.d.f.) was determined from $e^{-\chi^{2} / 2}$, using the $\chi^{2}$ function of the fit. The p.d.f. value has been calculated for each value of $|\Delta \Gamma| / \Gamma$ with all other fit parameters left free. The quoted statistical error of 0.30 on $|\Delta \Gamma| / \Gamma$ is the $68 \%$ confidence level (C.L.) upper limit value. At the $95 \%$ C.L., $|\Delta \Gamma| / \Gamma$ values larger than 0.55 are excluded.

The results for $\left\langle\tau_{\mathrm{B}}^{\mathrm{u}, \mathrm{d}}\right\rangle$ and $\tau\left(\mathrm{B}^{+}\right) / \tau\left(\mathrm{B}_{\mathrm{d}}^{0}\right)$ can also be given in terms of the $\mathrm{B}^{+}$and $\mathrm{B}_{\mathrm{d}}^{0}$ lifetimes, $\tau\left(\mathrm{B}^{+}\right)$and $\tau\left(\mathrm{B}_{\mathrm{d}}^{0}\right)$. The central values with the statistical errors are:

$$
\begin{aligned}
\tau\left(\mathrm{B}^{+}\right) & =1.66 \pm 0.06 \mathrm{ps} \\
\tau\left(\mathrm{B}_{\mathrm{d}}^{0}\right) & =1.52 \pm 0.06 \mathrm{ps} .
\end{aligned}
$$

The fit variables $\tau\left(\mathrm{B}^{+}\right)$and $\tau\left(\mathrm{B}_{\mathrm{d}}^{0}\right)$ are strongly correlated with a correlation coefficient of -0.78 . In the former case of $\left\langle\tau_{\mathrm{B}}^{\mathrm{u}, \mathrm{d}}\right\rangle$ and $\tau\left(\mathrm{B}^{+}\right) / \tau\left(\mathrm{B}_{\mathrm{d}}^{0}\right)$, the correlation is negligible.

The limit calculation has also been performed for a different definition of the measured $\mathrm{B}_{\mathrm{s}}^{0}$ lifetime $\tau\left(\mathrm{B}_{\mathrm{s}}^{0}\right)$. In the fit shown above, the average lifetime $\tau\left(\mathrm{B}_{\mathrm{s}}^{0}\right)$ was constrained to the measured value of $\tau\left(\mathrm{B}_{\mathrm{s}}^{0}\right)=1.49 \pm 0.06 \mathrm{ps}$ [11]. However, the individual measurements [9] do not determine $\tau\left(\mathrm{B}_{\mathrm{s}}^{0}\right)$ if $|\Delta \Gamma| / \Gamma \neq 0$. If a decay time distribution consisting of two distinct lifetimes 
$\tau_{1}$ and $\tau_{2}$ is fitted by a single exponential decay law, the fit result for the average lifetime lies between:

$$
\hat{\tau} \equiv 1 / \Gamma \quad \text { and } \quad \tau \equiv \frac{\left(\tau_{1}+\tau_{2}\right)}{2}=\frac{1 / \Gamma}{1-(\Delta \Gamma / 2 \Gamma)^{2}},
$$

with $\Gamma$ defined as the average decay rate $\Gamma \equiv\left(\Gamma_{1}+\Gamma_{2}\right) / 2$. The actual fitted value depends on the fit region, but is always between $\hat{\tau}$ and $\tau$. In order to account for this circumstance, the fit has been repeated with the definitions of $\tau_{1}$ and $\tau_{2}$ as:

$$
\tau_{1,2}=\frac{\hat{\tau}\left(\mathrm{B}_{\mathrm{s}}^{0}\right)}{1 \pm \Delta \Gamma / 2 \Gamma}
$$

instead of (5) and assuming that $\hat{\tau}\left(\mathrm{B}_{\mathrm{s}}^{0}\right)=1 / \Gamma\left(\mathrm{B}_{\mathrm{s}}^{0}\right)$ is the measured quantity. The upper limit on $|\Delta \Gamma| / \Gamma$ from this fit is better than with the original assumption, namely: $|\Delta \Gamma| / \Gamma<$ 0.45 (95\% C.L.). The first fit result of $|\Delta \Gamma| / \Gamma<0.55$ at $95 \%$ C.L. has been chosen as the statistical upper limit on $|\Delta \Gamma| / \Gamma$.

\section{Systematic Uncertainties}

The systematic error analysis applied to our previous measurement of the average b lifetime [5] has been repeated and complemented by the specific uncertainties of a charge-sensitive lifetime measurement. The results are summarised in Table 2. The systematic errors on the $\mathrm{B}_{\mathrm{s}}^{0}$ decay rate difference $|\Delta \Gamma| / \Gamma$, the lifetimes $\tau\left(\mathrm{B}^{+}\right), \tau\left(\mathrm{B}_{\mathrm{d}}^{0}\right)$ and the lifetime ratio $\tau\left(\mathrm{B}^{+}\right) / \tau\left(\mathrm{B}_{\mathrm{d}}^{0}\right)$ are shown.

The fitted value for $|\Delta \Gamma| / \Gamma$ is at the boundary of the physically allowed region. In order to determine the systematic error on $|\Delta \Gamma| / \Gamma$ properly, the fitted $|\Delta \Gamma| / \Gamma$ value in the data has been shifted to 0.3 by the use of the Monte Carlo. The data impact parameter distributions have been weighted by the ratio of the Monte Carlo distributions for $|\Delta \Gamma| / \Gamma=0.3$ and $|\Delta \Gamma| / \Gamma=0.0$. The error analysis has then been performed with this modified data sample.

The systematic error due to the tracking efficiency was derived from a comparison of the track multiplicity in hadronic events between data and Monte Carlo. The Monte Carlo distributions have been tuned to the data and the effect from this correction was taken as the systematic error. On average, about 0.1 tracks have been removed per event. For the uncertainty from the tracking resolution, the effects of calibration and alignment uncertainties have been simulated in the Monte Carlo and the corresponding changes in the fit parameters have been assigned as systematic errors. The negative impact parameter distributions in data and Monte Carlo have been used to determine correction parameters for the Monte Carlo impact parameter resolution. The uncertainty in these parameters has been translated into an uncertainty of the fit variables. This technique was model dependent since the source of the discrepancy was not known. Our standard fit procedure used two Gaussian convolution functions with different parameters for $b$ and non-b events. Other models with different assumptions have been compared with the standard fit. First, it was assumed that the correction is flavour-independent, i.e. the parameters were assumed to be equal for $b$ and non-b events. Second, the extreme cases have been considered where either the correction would apply only to b or non-b events, respectively. Finally, the standard fit procedure with two Gaussian functions has been extended to three Gaussian functions. The changes in the fit variables are collected in the line labelled "Resolution model" in Table 2. The largest detector uncertainties on the fit variables, in particular on $|\Delta \Gamma| / \Gamma$, were due to this uncertainty in the tracking resolution.

The normalisation of $b \bar{b}$ and $c \bar{c}$ events has been performed according to the LEP measurements [18]. The errors shown in Table 2 for the $\mathrm{b}$ quark fragmentation correspond to a 
range of the average $\mathrm{b}$ hadron energy of $\left\langle x_{E}\right\rangle_{b}=0.709 \pm 0.004$ (scaled to the beam energy) within the Peterson fragmentation model [19]. This parameter range refers to our previous measurement [5]. The transverse fragmentation has been studied by searching for differences in the transverse momentum distribution of tracks with respect to the jet axis. The tails of the $\mathrm{p}_{\perp}$ distribution have been found to be more pronounced in the data than in the Monte Carlo. The effect obtained from reweighting the tracks to the data $\mathrm{p}_{\perp}$ distribution has been assigned as a systematic error. The average charged b decay multiplicity was adjusted to our previous result of $\left\langle n_{b}\right\rangle=4.90 \pm 0.12$ [5]. The branching ratios $\mathcal{B}\left(\mathrm{B}^{0,+} \rightarrow \mathrm{D}^{-} \mathrm{X}\right), \mathcal{B}\left(\mathrm{B}^{0,+} \rightarrow \mathrm{D}_{\mathrm{s}}^{-} \mathrm{X}\right)$ and $\mathcal{B}\left(\mathrm{B}_{\mathrm{s}}^{0} \rightarrow \mathrm{D}_{\mathrm{s}}^{-} \mathrm{X}\right)$ (and their respective charge conjugates) have been varied by the uncertainty in the current world averages [17]. The fraction of $\mathrm{B}$ decays producing two $\mathrm{D}$ hadrons was assumed to be $0.15 \pm 0.05$. The fractions of $\mathrm{D}$ hadrons in $\mathrm{c} \overline{\mathrm{c}}$ events were varied according to reference [20]. The systematic uncertainties due to physics modelling were dominated by the uncertainties in the $\mathrm{B} \rightarrow \mathrm{D}$ decay chain.

Uncertainties in the charge measurement have also been studied. The cut on the secondary vertex charge $Q_{S V}$ influences the b hadron purities in the various subsamples. The $\mathrm{B}_{\mathrm{d}}^{0}$ and $\mathrm{B}_{\mathrm{s}}^{0}$ purities in the neutral sample (see Table 1) have been varied by $5 \%$ of the predicted enhancement with respect to the intrinsic purities. Simultaneously, the $\mathrm{B}_{\mathrm{d}}^{0}$ and $\mathrm{B}_{\mathrm{s}}^{0}$ purities in the other subsamples have been varied, conserving the total number of $\mathrm{B}_{\mathrm{d}}^{0}$ and $\mathrm{B}_{\mathrm{s}}^{0}$ mesons. The corresponding errors on the fit variables are given in the line labelled " $\mathrm{B}^{+} / \mathrm{B}^{0}$ separation" in Table 2 .

The uncertainty related to the limited knowledge of the lifetimes and production fractions of $\mathrm{B}_{\mathrm{s}}^{0}$ and $\Lambda_{\mathrm{b}}$ hadrons is already included in the statistical uncertainty due to their use as constrained fit parameters. In order to quantify the systematic content in the statistical errors of $|\Delta \Gamma| / \Gamma, \tau\left(\mathrm{B}^{+}\right), \tau\left(\mathrm{B}_{\mathrm{d}}^{0}\right)$ and $\tau\left(\mathrm{B}^{+}\right) / \tau\left(\mathrm{B}_{\mathrm{d}}^{0}\right)$ the fit has been repeated each time fixing a certain parameter. The quadratic differences between the original statistical errors when all fit variables were varied and these errors have been used as a measure of their contributions to the errors. The numbers are shown in Table 3. All contributions were small compared to the statistical errors.

A cross check has been performed by determining whether the fit is compatible with a zero value of $\Delta \Gamma$ for $\mathrm{B}^{+}$hadrons. A fit for a lifetime difference of $\mathrm{B}^{+}$mesons gave the result $|\Delta \Gamma| / \Gamma=0.00_{-0.00}^{+0.18}$, corresponding a $95 \%$ C.L. upper limit of $|\Delta \Gamma| / \Gamma<0.33$.

The same fit as performed on the impact parameter distributions has been applied to the decay length distributions. In this way, possible systematic uncertainties that affect the impact parameter distribution differently from the decay length distribution have been cross checked. The differences in the fit results were much smaller than their statistical errors.

\section{Final Results}

The combination of the statistical and systematic errors on $|\Delta \Gamma| / \Gamma$ has been performed as follows. The total systematic error of 0.21 has been scaled by a factor of 1.8 , the ratio of the statistical $95 \%$ C.L. and $68 \%$ C.L. limits, giving 0.38. This has been added in quadrature to the statistical upper limit of 0.55 , resulting in the combined $95 \%$ C.L. upper limit on $|\Delta \Gamma| / \Gamma$ of:

$$
|\Delta \Gamma| / \Gamma<0.67 \quad \text { (95\% C.L.) } .
$$

This result improves upon an upper limit on $|\Delta \Gamma| / \Gamma$ that has been reported recently by the CDF Collaboration [21]. 
The final result for the lifetime ratio $\tau\left(\mathrm{B}^{+}\right) / \tau\left(\mathrm{B}_{\mathrm{d}}^{0}\right)$ is:

$$
\tau\left(\mathrm{B}^{+}\right) / \tau\left(\mathrm{B}_{\mathrm{d}}^{0}\right)=1.09 \pm 0.07 \pm 0.03 .
$$

Using this ratio and $\left\langle\tau_{\mathrm{B}}^{\mathrm{u}, \mathrm{d}}\right\rangle$, and evaluating the systematic errors for the $\mathrm{B}^{+}$and $\mathrm{B}_{\mathrm{d}}^{0}$ lifetimes, we obtain:

$$
\begin{aligned}
\tau\left(\mathrm{B}^{+}\right) & =1.66 \pm 0.06 \pm 0.03 \mathrm{ps}, \\
\tau\left(\mathrm{B}_{\mathrm{d}}^{0}\right) & =1.52 \pm 0.06 \pm 0.04 \mathrm{ps} .
\end{aligned}
$$

The correlation coefficient between $\tau\left(\mathrm{B}^{+}\right)$and $\tau\left(\mathrm{B}_{\mathrm{d}}^{0}\right)$, including their systematic errors, is -0.52 . The results for $\tau\left(\mathrm{B}^{+}\right)$and $\tau\left(\mathrm{B}_{\mathrm{d}}^{0}\right)$ are in agreement with other measurements [22].

\section{Discussion}

In the Standard Model, the ratio $\Delta \Gamma_{\mathrm{B}_{\mathrm{s}}^{0}} / \Delta \mathrm{M}_{\mathrm{B}_{\mathrm{s}}^{0}}$ is given by a kinematical factor $\sim\left(m_{b} / m_{t}\right)^{2}$ and by QCD corrections [8]:

$$
\frac{\Delta \Gamma_{\mathrm{B}_{\mathrm{s}}^{0}}}{\Delta \mathrm{M}_{\mathrm{B}_{\mathrm{s}}^{0}}}=(5.6 \pm 2.6) \times 10^{-3} .
$$

The upper limit on $(\Delta \Gamma / \Gamma)_{\mathrm{B}_{\mathrm{s}}^{0}}$ can thus be converted into an upper limit on the $\mathrm{B}_{\mathrm{s}}^{0}$ meson oscillation frequency:

$$
\left(\frac{\Delta \mathrm{M}}{\Gamma}\right)_{\mathrm{B}_{\mathrm{s}}^{0}}<120 \quad(95 \% \text { C.L. }) .
$$

Here we used the central value for the theoretical prediction of $\Delta \Gamma_{\mathrm{B}_{\mathrm{s}}^{0}} / \Delta \mathrm{M}_{\mathrm{B}_{\mathrm{s}}^{0}}$. Measurements of $\Delta \mathrm{M}_{\mathrm{B}_{\mathrm{s}}^{0}}$ and $\Delta \Gamma_{\mathrm{B}_{\mathrm{s}}^{0}}$ are complementary to each other. Oscillation measurements are sensitive to values of $\Delta \mathrm{M}_{\mathrm{B}_{\mathrm{s}}^{0}}$ below an upper limit value determined by the experimental vertex resolution. In contrast to this, the $\Delta \Gamma_{\mathrm{B}_{\mathrm{s}}^{0}}$ measurement is applicable for values of $\Delta \mathrm{M}_{\mathrm{B}_{\mathrm{s}}^{0}}$ above a lower limit value. Fast $\mathrm{B}_{\mathrm{s}}^{0}$ oscillations with an oscillation frequency much higher than the present resolution can thus be resolved by a measurement of $\Delta \Gamma_{\mathrm{B}_{\mathrm{s}}^{0}}$.

An upper limit on $\Delta \mathrm{M}_{\mathrm{B}_{\mathrm{s}}^{0}}$, together with a measurement of $\Delta \mathrm{M}_{\mathrm{B}_{\mathrm{d}}^{0}}$, can be used to determine a lower limit on the ratio of CKM matrix elements $\left|V_{t d} / V_{t s}\right|$. Using our measured value for $\Delta \mathrm{M}_{\mathrm{B}_{\mathrm{d}}^{0}}[3]$ we obtain ${ }^{4)}$ :

$$
\left|\frac{\mathrm{V}_{\mathrm{td}}}{\mathrm{V}_{\mathrm{ts}}}\right|>0.09 \quad \text { (95\% C.L.) }
$$

This lower limit for $\left|\mathrm{V}_{\mathrm{td}} / \mathrm{V}_{\mathrm{ts}}\right|$ is comparable with the limit derived from the unitarity constraint of the CKM matrix of $\left|\mathrm{V}_{\mathrm{td}} / \mathrm{V}_{\mathrm{ts}}\right|>0.1$ at $90 \%$ C.L. [17].

\section{Acknowledgements}

We wish to express our gratitude to the CERN accelerator divisions for the excellent performance of the LEP machine. We acknowledge the effort of all engineers and technicians who have participated in the construction and maintenance of the experiment.

\footnotetext{
${ }^{4)}$ Beside $\Delta \Gamma_{\mathrm{B}_{\mathrm{s}}^{0}} / \Delta \mathrm{M}_{\mathrm{B}_{\mathrm{s}}^{0}}=5.6 \times 10^{-3}[8]$, we used here $\left(\sqrt{\mathrm{B}_{\mathrm{s}}} \mathrm{f}_{\mathrm{s}}\right) /\left(\sqrt{\mathrm{B}_{\mathrm{d}}} \mathrm{f}_{\mathrm{d}}\right)=1.16[8], \Delta \mathrm{M}_{\mathrm{B}_{\mathrm{d}}^{0}}=0.444 \mathrm{ps}^{-1}[3]$ and $\tau\left(\mathrm{B}_{\mathrm{s}}^{0}\right)=1.49 \mathrm{ps}$ [11]. To scale our lower limit on $\left|\mathrm{V}_{\mathrm{td}} / \mathrm{V}_{\mathrm{ts}}\right|$ to other values of these parameters, the dependence is: $\left|\mathrm{V}_{\mathrm{td}} / \mathrm{V}_{\mathrm{ts}}\right| \propto\left(\Delta \Gamma_{\mathrm{B}_{\mathrm{s}}^{0}} / \Delta \mathrm{M}_{\mathrm{B}_{\mathrm{s}}^{0}}\right)^{1 / 2}\left(\sqrt{\mathrm{B}_{\mathrm{s}}} \mathrm{f}_{\mathrm{s}}\right) /\left(\sqrt{\mathrm{B}_{\mathrm{d}}} \mathrm{f}_{\mathrm{d}}\right)\left(\Delta \mathrm{M}_{\mathrm{B}_{\mathrm{d}}^{0}} \tau\left(\mathrm{B}_{\mathrm{s}}^{0}\right)\right)^{1 / 2}$.
} 


\section{References}

[1] UA1 Collaboration, C. Albajar et al., Phys. Lett. B 186 (1987) 247;

ARGUS Collaboration, H. Albrecht et al., Phys. Lett. B 192 (1987) 245;

ARGUS Collaboration, H. Albrecht et al., Phys. Lett. B 324 (1994) 249;

CLEO Collaboration, J. Bartelt et al., Phys. Rev. D 50 (1994) 43;

ALEPH Collaboration, D. Decamp et al., Phys. Lett. B 284 (1992) 177;

DELPHI Collaboration, P. Abreu et al., Phys. Lett. B 332 (1994) 488;

L3 Collaboration, M. Acciarri et al., Phys. Lett. B 335 (1994) 542;

OPAL Collaboration, R. Akers et al., Z. Phys. C 60 (1993) 199.

[2] ALEPH Collaboration, D. Buskulic et al., Z. Phys. C 75 (1997) 397;

DELPHI Collaboration P. Abreu et al., CERN-PPE/97-51, to be published in E. Phys. J. C.;

L3 Collaboration, M. Acciarri et al., Phys. Lett. B 383 (1996) 487;

OPAL Collaboration, R. Akers et al., Z. Phys. C 72 (1996) 377;

OPAL Collaboration, R. Akers et al., Z. Phys. C 76 (1997) 401;

OPAL Collaboration, R. Akers et al., Z. Phys. C 76 (1997) 417.

[3] L3 Collaboration M. Acciarri et al., CERN-EP/98-028, to be published in E. Phys. J. C.

[4] ALEPH Collaboration, D. Buskulic et al., Phys. Lett. B 314 (1993) 459;

ALEPH Collaboration, D. Buskulic et al., Phys. Lett. B 369 (1996) 151;

DELPHI Collaboration, P. Abreu et al., Z. Phys. C 63 (1994) 3;

DELPHI Collaboration, P. Abreu et al., Phys. Lett. B 377 (1996) 195;

L3 Collaboration, O. Adriani et al., Phys. Lett. B 317 (1993) 474;

OPAL Collaboration, P.D. Acton et al., Z. Phys. C 60 (1993) 217;

OPAL Collaboration, P.D. Acton et al., Z. Phys. C 73 (1997) 397;

SLD Collaboration, K. Abe et al., Phys. Rev. Lett. 75 (1997) 3624;

CDF Collaboration, F. Abe et al., Phys. Rev. Lett. 71 (1993) 3421.

[5] L3 Collaboration, M. Acciarri et al., Phys. Lett. B 416 (1998) 220.

[6] M. Shifman, N.G. Uraltsev and A. Vainshtein, Phys. Rev. D 51 (1995) 2217.

[7] J.S. Hagelin, Nucl. Phys. B 193 (1981) 123;

R. Aleksan et al., Phys. Lett. B 316, (1993) 567.

[8] I. Dunietz, Phys. Rev. D 52 (1995) 3048;

M. Beneke, G. Buchalla and I. Dunietz, Phys. Rev. D 54 (1996) 4419.

[9] ALEPH Collaboration, D. Buskulic et al., Phys. Lett. B 377 (1996) 205;

ALEPH Collaboration, D. Buskulic et al., Phys. Lett. B 384 (1996) 449;

DELPHI Collaboration, P. Abreu et al., Z. Phys. C 71 (1996) 11;

OPAL Collaboration, R. Akers et al., Phys. Lett. B 350 (1995) 273;

OPAL Collaboration, R. Akers et al., CERN-PPE/97-095, to be published in Z. Phys. C.;

CDF Collaboration, F. Abe et al., Phys. Rev. Lett. 74 (1995) 4988;

CDF Collaboration, F. Abe et al., Phys. Rev. Lett. 77 (1996) 1945;

CDF Collaboration, F. Abe et al., Phys. Rev. D 57 (1998) 5382. 
[10] ALEPH Collaboration, D. Buskulic et al., Phys. Lett. B 357 (1995) 685;

DELPHI Collaboration, P. Abreu et al., Z. Phys. C 68 (1995) 375;

OPAL Collaboration, R. Akers et al., Phys. Lett. B 353 (1995) 402;

OPAL Collaboration, R. Akers et al., Z. Phys. C 69 (1996) 195;

CDF Collaboration, F. Abe et al., Phys. Rev. Lett. 77 (1996) 1439.

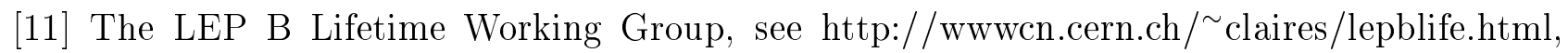
For a recent overview see, F. Muheim, talk presented at Int. Conf. on High-energy Phys. (ICHEP 97), Jerusalem.

[12] The L3 Collaboration, B. Adeva et al., Nucl. Instr. and Meth. A 289 (1990) 35;

M. Chemarin et al., Nucl. Instr. and Meth. A 349 (1994) 345;

A. Adam et al., CERN-PPE/96-097;

I.C. Brock et al., Nucl. Instr. and Meth. A 381 (1996) 236;

L3 Collaboration, M. Acciarri et al., Nucl. Instr. and Meth. A 351 (1994) 300.

[13] L3 Collaboration, M. Acciarri et al., Z. Phys. C 62 (1994) 551.

[14] S. Petrak, PhD thesis, Humboldt-Universität Berlin, 1998, unpublished.

[15] T. Sjöstrand, Comp. Phys. Comm. 39 (1986) 347;

T. Sjöstrand and M. Bengtsson, Comp. Phys. Comm. 43 (1987) $367 .$.

[16] The L3 detector simulation is based on GEANT Version 3.15.

R. Brun et al., GEANT 3, CERN-DD/EE/84-1 (Revised), 1987.

The GHEISHA program (H. Fesefeldt, RWTH Aachen Report PITHA 85/02 (1985))

is used to simulate hadronic interactions.

[17] Particle Data Group, C. Caso et al., E. Phys. J C3 (1998) 1.

[18] The LEP Experiments: ALEPH, DELPHI, L3, OPAL, CERN-PPE/97-154.

[19] C. Peterson, D. Schlatter, I. Schmitt and P.M. Zerwas, Phys. Rev. D 27 (1983) 105.

[20] The LEP Experiments: ALEPH, DELPHI, L3, OPAL, Nucl. Inst. Meth. A 378 (1996) 101.

[21] CDF Collaboration, F. Abe et al., Fermilab-Pub-98/172-E, to be published in Phys. Rev. D.

[22] ALEPH Collaboration, D. Buskulic et al., Z. Phys. C 71 (1996) 31;

DELPHI Collaboration, P. Abreu et al., Z. Phys. C 68 (1995) 13;

DELPHI Collaboration, W. Adam et al., Z. Phys. C 68 (1995) 363;

DELPHI Collaboration, P. Abreu et al., Z. Phys. C 74 (1997) 19;

OPAL Collaboration, R. Akers et al., Z. Phys. C 67 (1995) 379;

CDF Collaboration, F. Abe et al., Phys. Rev. D 57 (1998) 5382;

CDF Collaboration, F. Abe et al., Phys. Rev. Lett. 76 (1996) 4462;

SLD Collaboration, K. Abe et al., Phys. Rev. Lett. 79 (1997) 590. 


\section{The L3 Collaboration:}

M.Acciarri ${ }^{27}$ O.Adriani ${ }^{16}$ M.Aguilar-Benitez ${ }^{26}$ S.Ahlen $^{11}{ }^{1}$ J.Alcaraz ${ }^{26}$ G.Alemanni ${ }^{22}$ J.Allaby, ${ }^{17}$ A.Aloisio ${ }^{29}$ M.G.Alviggi ${ }^{29}$ G.Ambrosi, ${ }^{19}$ H.Anderhub, ${ }^{48}$ V.P.Andreev ${ }^{37}$ T.Angelescu, ${ }^{13}$ F.Anselmo, ${ }^{9}$ A.Arefiev ${ }^{28}$ T.Azemoon, ${ }^{3}$ T.Aziz, ${ }^{10}$ P.Bagnaia, ${ }^{36}$ L.Baksay, ${ }^{43}$ R.C.Ball, ${ }^{3}$ S.Banerjee, ${ }^{10}$ Sw.Banerjee, ${ }^{10}$ K.Banicz, ${ }^{45}$ A.Barczyk, ${ }^{48,46}$ R.Barillère ${ }^{17}$ L.Barone ${ }^{36}$ P.Bartalini ${ }^{22}$ A.Baschirotto, ${ }^{27}$ M.Basile, ${ }^{9}$ R.Battiston ${ }^{33}$ A.Bay ${ }^{22}$ F.Becattini, ${ }^{16}$ U.Becker, ${ }^{15}$ F.Behner, ${ }^{48}$ J.Berdugo, ${ }^{26}$ P.Berges ${ }^{15}$ B.Bertucci ${ }^{33}$ B.L.Betev, ${ }^{48}$ S.Bhattacharya ${ }^{10}$ M.Biasini ${ }^{33}$ A.Biland, ${ }^{48}$ G.M.Bilei ${ }^{33}$ J.J.Blaising, ${ }^{4}$ S.C.Blyth ${ }^{34}$ G.J.Bobbink ${ }^{2}$ R.Bock, ${ }^{1}$ A.Böhm, ${ }^{1}$ L.Boldizsar, ${ }^{14}$ B.Borgia,${ }^{17,36}$ D.Bourilkov, ${ }^{48}$ M.Bourquin, ${ }^{19}$ D.Boutigny, ${ }^{4}$ S.Braccini, ${ }^{19}$ J.G.Branson ${ }^{39}$ V.Brigljevic, ${ }^{48}$ I.C.Brock, ${ }^{34}$ A.Buffini ${ }^{16}$ A.Buijs, ${ }^{44}$ J.D.Burger, ${ }^{15}$ W.J.Burger ${ }^{33}$ J.Busenitz, ${ }^{43}$ X.D.Cai ${ }^{15}$ M.Campanelli, ${ }^{48}$ M.Capell $^{15}{ }^{\text {G.Cara Romeo, }}{ }^{9}$ G.Carlino, ${ }^{29}$ A.M.Cartacci ${ }^{16}$ J.Casaus, ${ }^{26}$ G.Castellini ${ }^{16}$ F.Cavallari ${ }^{36}$ N.Cavallo ${ }^{29}$ C.Cecchi ${ }^{19}$ M.Cerrada ${ }^{26}$ F.Cesaroni ${ }^{23}$ M.Chamizo, ${ }^{26}$ Y.H.Chang, ${ }^{50}$ U.K.Chaturvedi, ${ }^{18}$ M.Chemarin ${ }^{25}$ A.Chen ${ }^{50}$ G.Chen, ${ }^{7}$ G.M.Chen, H.F.Chen ${ }^{20}$ H.S.Chen, ${ }^{7}$ M.Chen, ${ }^{15}$ G.Chiefari ${ }^{29}$ C.Y.Chien, ${ }^{5}$ L.Cifarelli ${ }^{38}$ F.Cindolo, C.Civinini ${ }^{16}{ }^{9}$ I.Clare, ${ }^{15}$ R.Clare, ${ }^{15}$ G.Coignet, ${ }^{4}$ A.P.Colijn, 2 N.Colino, ${ }^{26}$ S.Costantini, ${ }^{8}$ F.Cotorobai ${ }^{13}$ B.de la Cruz ${ }^{26}$ A.Csilling ${ }^{14}$ T.S.Dai ${ }^{15}$ R.D'Alessandro, ${ }^{16}$ R.de Asmundis, ${ }^{29}$ A.Degré, ${ }^{4}$ K.Deiters, ${ }^{46}$ P.Denes, ${ }^{35}$ F.DeNotaristefani, ${ }^{36}$ M.Diemoz, ${ }^{36}$ D.van Dierendonck, F.Di Lodovico, ${ }^{48}$ C.Dionisi, ${ }^{1,36}$ M.Dittmar ${ }^{48}$ A.Dominguez, ${ }^{39}$ A.Doria, ${ }^{29}$ M.T.Dova, ${ }^{18, \sharp}$ E.Drago, ${ }^{29}$ D.Duchesneau, ${ }^{4}$ P.Duinker, ${ }^{2}$ I.Duran,${ }^{40}$ S.Easo ${ }^{33}$ H.El Mamouni ${ }^{25}$ A.Engler ${ }^{34}$ F.J.Eppling, ${ }^{15}$ F.C.Erné ${ }^{2}$ P.Extermann ${ }^{19}$ M.Fabre ${ }^{46}$ R.Faccini ${ }^{36}$ M.A.Falagan ${ }^{26}$ S.Falciano ${ }^{36}$ A.Favara ${ }^{16}{ }^{16}$ J.Fay, ${ }^{25}$ O.Fedin ${ }^{37}$ M.Felcini ${ }^{48}$ T.Ferguson, ${ }^{34}$ F.Ferroni ${ }^{36}$ H.Fesefeldt, ${ }^{1}$ E.Fiandrini, ${ }^{33}$ J.H.Field ${ }^{19}$ F.Filthaut ${ }^{17}$ P.H.Fisher ${ }^{15}$ I.Fisk ${ }^{39}$ G.Forconi ${ }^{15}$ L.Fredj ${ }^{19}$ K.Freudenreich, ${ }^{48}$ C.Furetta ${ }^{27}$ Yu.Galaktionov, ${ }^{28,15}$ S.N.Ganguli, ${ }^{10}$ P.Garcia-Abia, ${ }^{6}$ M.Gataullin ${ }^{32}$ S.S.Gau, ${ }^{12}$ S.Gentile, ${ }^{36}$ J.Gerald ${ }^{5}$ N.Gheordanescu, ${ }^{13}$ S.Giagu, ${ }^{36}$ S.Goldfarb, ${ }^{22}$ J.Goldstein, ${ }^{11}$ Z.F.Gong, ${ }^{20}$ A.Gougas, G.Gratta, ${ }^{32}$ M.W.Gruenewald, ${ }^{8}$ R.van Gulik, V.K.Gupta ${ }^{35}$ A.Gurtu ${ }^{10}$ L.J.Gutay, ${ }^{45}$ D.Haas, ${ }^{6}$ B.Hartmann, A.Hasan ${ }^{30}$ D.Hatzifotiadou, ${ }^{9}$ T.Hebbeker, ${ }^{8}$ A.Hervé ${ }^{17}$ P.Hidas, ${ }^{14}$ J.Hirschfelder, ${ }^{34}$ W.C.van Hoek ${ }^{31}$ H.Hofer ${ }^{48}$ H.Hoorani ${ }^{34}$ S.R.Hou ${ }^{50}$ G.Hu, ${ }^{5}$ I.Iashvili, ${ }^{47}$ B.N.Jin, ${ }^{7}$ L.W.Jones, ${ }^{3}$ P.de Jong, ${ }^{17}$ I.Josa-Mutuberria, ${ }^{26}$ A.Kasser, ${ }^{22}$ R.A.Khan ${ }^{18}$ D.Kamrad, ${ }^{47}$ J.S.Kapustinsky, ${ }^{24}$ Y.Karyotakis, ${ }^{4}$ M.Kaur, ${ }^{18, \diamond}$ M.N.Kienzle-Focacci, ${ }^{19}$ D.Kim, ${ }^{36}$ D.H.Kim ${ }^{42}$ J.K.Kim ${ }^{42}$ S.C.Kim ${ }^{42}$ W.W.Kinnison, ${ }^{24}$ A.Kirkby ${ }^{32}$ D.Kirkby, ${ }^{32}$ J.Kirkby, ${ }^{17}$ D.Kiss,${ }^{14}$ W.Kittel ${ }^{31}$ A.Klimentov ${ }^{15,28}$ A.C.König, ${ }^{31}$ A.Kopp ${ }^{47}$ I.Korolko, ${ }^{28}$ V.Koutsenko, ${ }^{15,28}$ R.W.Kraemer, ${ }^{34}$ W.Krenz, ${ }^{1}$ A.Kunin, ${ }^{15,28}$ P.Lacentre ${ }^{47, b, \sharp}$ P.Ladron de Guevara ${ }^{26}$ G.Landi ${ }^{16}$ C.Lapoint ${ }^{15}$ K.Lassila-Perini ${ }^{48}$ P.Laurikainen ${ }^{21}$ A.Lavorato, ${ }^{38}$ M.Lebeau, ${ }^{17}$ A.Lebedev, ${ }^{15}$ P.Lebrun ${ }^{25}$ P.Lecomte, ${ }^{48}$ P.Lecoq ${ }^{17}$ P.Le Coultre ${ }^{48}$ H.J.Lee ${ }^{8}$ C.Leggett, ${ }^{3}$ J.M.Le Goff ${ }^{17}$ R.Leiste ${ }^{47}$ E.Leonardi, ${ }^{36}$ P.Levtchenko, ${ }^{37}$ C.Li ${ }^{20}$ C.H.Lin ${ }^{50}$ W.T.Lin ${ }^{50}$ F.L.Linde,${ }^{2,17}$ L.Lista ${ }^{29}$ Z.A.Liu, W.Lohmann, ${ }^{47}$ E.Longo, ${ }^{36}$ W.Lu, ${ }^{32}$ Y.S.Lu, ${ }^{7}$ K.Lübelsmeyer, ${ }^{1}$ C.Luci ${ }^{17,36}$ D.Luckey, ${ }^{15}$ L.Luminari ${ }^{36}$ W.Lustermann ${ }^{48}$ W.G.Ma ${ }^{20}$ M.Maity, ${ }^{10}$ G.Majumder ${ }^{10}$ L.Malgeri ${ }^{17}$ A.Malinin, ${ }^{28}$ C.Maña ${ }^{26}$ D.Mangeol, ${ }^{31}$ P.Marchesini, ${ }^{48}$ G.Marian, ${ }^{43}$, A.Marin, ${ }^{11}$ J.P.Martin ${ }^{25}$ F.Marzano, ${ }^{36}$ G.G.G.Massaro, K.Mazumdar, ${ }^{10}$ S.Mele, ${ }^{17}$ L.Merola, ${ }^{29}$ M.Meschini, ${ }^{16}$ W.J.Metzger, ${ }^{31}$ M.von der Mey, ${ }^{1}$ Y.Mi, ${ }^{22}$ D.Migani, ${ }^{9}$ A.Mihul, ${ }^{13}$ A.J.W.van Mil ${ }^{31}$ H.Milcent, ${ }^{17}$ G.Mirabellii, ${ }^{36}$ J.Mnich, ${ }^{17}$ P.Molnar, ${ }^{8}$ B.Monteleoni ${ }^{16}$ R.Moore, ${ }^{3}$ T.Moulik, ${ }^{10}$ R.Mount ${ }^{32}$ G.S.Muanza ${ }^{25}$ F.Muheim ${ }^{19}$ A.J.M.Muijs, ${ }^{2}$ S.Nahn, ${ }^{15}$ M.Napolitano, ${ }^{29}$ F.Nessi-Tedaldi, ${ }^{48}$ H.Newman ${ }^{32}$ T.Niessen, A.Nippe ${ }^{22}$ A.Nisati ${ }^{36}$ H.Nowak ${ }^{47}$ Y.D.OH ${ }^{42}$ G.Organtini ${ }^{36}$ R.Ostonen ${ }^{21}$ C.Palomares ${ }^{26}$ D.Pandoulas, ${ }^{1}$ S.Paoletti ${ }^{36,17}$ P.Paolucci ${ }^{29}$ H.K.Park ${ }^{34}$ I.H.Park ${ }^{42}$ G.Pascale, ${ }^{36}$ G.Passaleva,${ }^{17}$ S.Patricelli ${ }^{29}{ }^{2}$ T.Paul ${ }^{12}$ M.Pauluzzi ${ }^{33}$ C.Paus ${ }^{17}$ F.Pauss ${ }^{48}$ D.Peach ${ }^{17}$ M.Pedace ${ }^{36}$ Y.J.Pei ${ }^{1}$ S.Pensotti ${ }^{27}$ D.Perret-Gallix, ${ }^{4}$ B.Petersen ${ }^{31}$ S.Petrak, ${ }^{8}$ A.Pevsner, ${ }^{5}$ D.Piccolo, ${ }^{29}$ M.Pieri, ${ }^{16}$ P.A.Piroué, ${ }^{35}$ E.Pistolesi ${ }^{27}$ V.Plyaskin ${ }^{28}$ M.Pohl $^{48}$ V.Pojidaev ${ }^{28,16}$ H.Postema, ${ }^{15}$ J.Pothier $^{17}$ N.Produit ${ }^{19}$ D.Prokofiev, ${ }^{37}$ J.Quartieri ${ }^{38}$ G.Rahal-Callot ${ }^{48}$ N.Raja, ${ }^{10}$ P.G.Rancoita ${ }^{27}$ M.Rattaggi, ${ }^{27}$ G.Raven ${ }^{39}$ P.Razis, ${ }^{30}$ D.Ren ${ }^{48}$ M.Rescigno, ${ }^{36}$ S.Reucroft, ${ }^{12}$ T.van Rhee, ${ }^{44}$ S.Riemann, ${ }^{47}$ K.Riles, ${ }^{3}$ O.Rind, ${ }^{3}$ A.Robohm, J.Rodin, ${ }^{43}$ B.P.Roe, ${ }^{3}$ L.Romero, ${ }^{26}$ S.Rosier-Lees, ${ }^{4}$ Ph.Rosselet ${ }^{22}$ S.Roth, ${ }^{1}$ J.A.Rubio, ${ }^{17}$ D.Ruschmeier, ${ }^{8}$ H.Rykaczewski, ${ }^{48}$ S.Sakar, ${ }^{36}$ J.Salicio, ${ }^{17}$ E.Sanchez ${ }^{26}$ M.P.Sanders ${ }^{31}$ M.E.Sarakinos ${ }^{21}$ G.Sauvage, C.Schäfer ${ }^{1}$ V.Schegelsky, ${ }^{37}$ S.Schmidt-Kaerst, D.Schmitz, ${ }^{1}$ M.Schneegans, ${ }^{4}$ N.Scholz, ${ }^{48}$ H.Schopper, ${ }^{49}$ D.J.Schotanus, ${ }^{31}$ J.Schwenke, G.Schwering, C.Sciacca, ${ }^{19}$ D.Sciarrino, ${ }^{19}$ L.Servoli, ${ }^{33}$ S.Shevchenko, ${ }^{32}$ N.Shivarov, ${ }^{41}$ V.Shoutko, ${ }^{28}$ J.Shukla ${ }^{24}$ E.Shumilov, ${ }^{28}$ A.Shvorob, ${ }^{32}$ T.Siedenburg, ${ }^{1}$ D.Son, ${ }^{42}$ V.Soulimov ${ }^{29}$ B.Smith, ${ }^{15}$ P.Spillantini ${ }^{16}$ M.Steuer, ${ }^{15}$ D.P.Stickland, ${ }^{35}$ H.Stone, ${ }^{35}$ B.Stoyanov, ${ }^{41}$ A.Straessner, ${ }^{1}$ K.Sudhakar, ${ }^{10}$ G.Sultanov, ${ }^{18}$ L.Z.Sun ${ }^{20}$ G.F.Susinno, ${ }^{19}$ H.Suter, ${ }^{48}$ J.D.Swain ${ }^{18}$ X.W.Tang, ${ }^{7}$ L.Tauscher, ${ }^{6}$ L.Taylor, ${ }^{12}$ C.Timmermans, ${ }^{31}$ Samuel C.C.Ting, ${ }^{15}$ S.M.Ting, ${ }^{15}$ S.C.Tonwar ${ }^{10}$ J.Tóth ${ }^{14}$ C.Tully ${ }^{35}$ K.L.Tung, ${ }^{7}$ Y.Uchida, ${ }^{15}$ J.Ulbrichtt, ${ }^{48}$ E.Valente ${ }^{36}$ G.Vesztergombi ${ }^{14}$ I.Vetlitsky $^{28}$ G.Viertel ${ }^{48}$ S.Villa, ${ }^{12}$ M.Vivargent, ${ }^{4}$ S.Vlachos, ${ }^{6}$ H.Vogel ${ }^{34}$ H.Vogt ${ }^{47}{ }^{4}$ I.Vorobiev, ${ }^{17,28}$ A.A.Vorobyov ${ }^{37}$ A.Vorvolakos ${ }^{30}$ M.Wadhwa, W.Wallraff, J.C.Wang, ${ }^{15}$ X.L.Wang, ${ }^{20}$ Z.M.Wang, ${ }^{20}$ A.Weber, ${ }^{1}$ S.X.Wu ${ }^{15}{ }^{15}$ S.Wynhoff, ${ }^{1}$ J.Xu, ${ }^{11}$ Z.Z.Xu ${ }^{20}$ B.Z.Yang, ${ }^{20}$ C.G.Yang, H.J.Yang, M.Yang, ${ }^{7}$ J.B.Ye, ${ }^{20}{ }^{7}$ S.C.Yeh ${ }^{51}$ J.M.You, ${ }^{34}$ An.Zalite, ${ }^{37}$ Yu.Zalite, ${ }^{37}$ P.Zemp ${ }^{48}$ Y.Zeng, Z.P.Zhang, ${ }^{20}$ B.Zhou, ${ }^{11}$ Y.Zhou, ${ }^{3}$ G.Y.Zhu, R.Y.Zhu, ${ }^{32}$ A.Zichichi, ${ }^{9,17}{ }^{78}$ F.Ziegler, ${ }^{47}$ G.Zilizi. ${ }^{43,}$ 
1 I. Physikalisches Institut, RWTH, D-52056 Aachen, FRG ${ }^{\S}$

III. Physikalisches Institut, RWTH, D-52056 Aachen, FRG ${ }^{\S}$

2 National Institute for High Energy Physics, NIKHEF, and University of Amsterdam, NL-1009 DB Amsterdam, The Netherlands

3 University of Michigan, Ann Arbor, MI 48109, USA

4 Laboratoire d'Annecy-le-Vieux de Physique des Particules, LAPP,IN2P3-CNRS, BP 110, F-74941 Annecy-le-Vieux CEDEX, France

5 Johns Hopkins University, Baltimore, MD 21218, USA

6 Institute of Physics, University of Basel, CH-4056 Basel, Switzerland

7 Institute of High Energy Physics, IHEP, 100039 Beijing, China ${ }^{\triangle}$

8 Humboldt University, D-10099 Berlin, FRG $^{\S}$

9 University of Bologna and INFN-Sezione di Bologna, I-40126 Bologna, Italy

10 Tata Institute of Fundamental Research, Bombay 400 005, India

11 Boston University, Boston, MA 02215, USA

12 Northeastern University, Boston, MA 02115, USA

13 Institute of Atomic Physics and University of Bucharest, R-76900 Bucharest, Romania

14 Central Research Institute for Physics of the Hungarian Academy of Sciences, H-1525 Budapest 114, Hungary ${ }^{\ddagger}$

15 Massachusetts Institute of Technology, Cambridge, MA 02139, USA

16 INFN Sezione di Firenze and University of Florence, I-50125 Florence, Italy

17 European Laboratory for Particle Physics, CERN, CH-1211 Geneva 23, Switzerland

18 World Laboratory, FBLJA Project, CH-1211 Geneva 23, Switzerland

19 University of Geneva, CH-1211 Geneva 4, Switzerland

20 Chinese University of Science and Technology, USTC, Hefei, Anhui 230 029, China $\triangle$

21 SEFT, Research Institute for High Energy Physics, P.O. Box 9, SF-00014 Helsinki, Finland

22 University of Lausanne, CH-1015 Lausanne, Switzerland

23 INFN-Sezione di Lecce and Universitá Degli Studi di Lecce, I-73100 Lecce, Italy

24 Los Alamos National Laboratory, Los Alamos, NM 87544, USA

25 Institut de Physique Nucléaire de Lyon, IN2P3-CNRS,Université Claude Bernard, F-69622 Villeurbanne, France

26 Centro de Investigaciones Energeticas, Medioambientales y Tecnologicas, CIEMAT, E-28040 Madrid, Spainb

27 INFN-Sezione di Milano, I-20133 Milan, Italy

28 Institute of Theoretical and Experimental Physics, ITEP, Moscow, Russia

29 INFN-Sezione di Napoli and University of Naples, I-80125 Naples, Italy

30 Department of Natural Sciences, University of Cyprus, Nicosia, Cyprus

31 University of Nijmegen and NIKHEF, NL-6525 ED Nijmegen, The Netherlands

32 California Institute of Technology, Pasadena, CA 91125, USA

33 INFN-Sezione di Perugia and Universitá Degli Studi di Perugia, I-06100 Perugia, Italy

34 Carnegie Mellon University, Pittsburgh, PA 15213, USA

35 Princeton University, Princeton, NJ 08544, USA

36 INFN-Sezione di Roma and University of Rome, "La Sapienza", I-00185 Rome, Italy

37 Nuclear Physics Institute, St. Petersburg, Russia

38 University and INFN, Salerno, I-84100 Salerno, Italy

39 University of California, San Diego, CA 92093, USA

40 Dept. de Fisica de Particulas Elementales, Univ. de Santiago, E-15706 Santiago de Compostela, Spain

41 Bulgarian Academy of Sciences, Central Lab. of Mechatronics and Instrumentation, BU-1113 Sofia, Bulgaria

42 Center for High Energy Physics, Adv. Inst. of Sciences and Technology, 305-701 Taejon, Republic of Korea

43 University of Alabama, Tuscaloosa, AL 35486, USA

44 Utrecht University and NIKHEF, NL-3584 CB Utrecht, The Netherlands

45 Purdue University, West Lafayette, IN 47907, USA

46 Paul Scherrer Institut, PSI, CH-5232 Villigen, Switzerland

47 DESY-Institut für Hochenergiephysik, D-15738 Zeuthen, FRG

48 Eidgenössische Technische Hochschule, ETH Zürich, CH-8093 Zürich, Switzerland

49 University of Hamburg, D-22761 Hamburg, FRG

50 National Central University, Chung-Li, Taiwan, China

51 Department of Physics, National Tsing Hua University, Taiwan, China

$\S$ Supported by the German Bundesministerium für Bildung, Wissenschaft, Forschung und Technologie

$\mp$ Supported by the Hungarian OTKA fund under contract numbers T019181, F023259 and T024011.

ๆ Also supported by the Hungarian OTKA fund under contract numbers T22238 and T026178.

b Supported also by the Comisión Interministerial de Ciencia y Technología.

\# Also supported by CONICET and Universidad Nacional de La Plata, CC 67, 1900 La Plata, Argentina.

Ł Supported by Deutscher Akademischer Austauschdienst.

$\diamond$ Also supported by Panjab University, Chandigarh-160014, India.

$\triangle$ Supported by the National Natural Science Foundation of China. 


\begin{tabular}{|c|c|c|c|c|c|c|}
\hline \multirow[t]{2}{*}{ Sample } & \multirow{2}{*}{$\begin{array}{l}\text { Number of } \\
\text { secondary } \\
\text { vertices }\end{array}$} & \multicolumn{2}{|c|}{ b quark purity } & \multicolumn{3}{|c|}{$\begin{array}{c}\text { b hadron purities } \\
\text { MC }\end{array}$} \\
\hline & & $\mathrm{MC}$ & Fit & $\mathrm{B}^{+}$ & $\mathrm{B}_{\mathrm{d}}^{0}$ & $\mathrm{~B}_{\mathrm{s}}^{0}$ \\
\hline Charged: $\begin{aligned} Q_{S V} & >0.5 \\
& l_{o p p}<2 \mathrm{~mm} \\
& l_{o p p}>2 \mathrm{~mm}\end{aligned}$ & $\begin{array}{r}319374 \\
72224 \\
\end{array}$ & $\begin{array}{r}16.8 \% \\
67.5 \% \\
\end{array}$ & $(67.6 \pm 0.5) \%$ & $44.3 \%$ & $36.7 \%$ & $9.6 \%$ \\
\hline 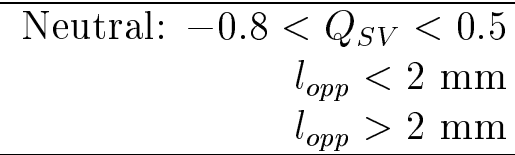 & $\begin{array}{r}310103 \\
66565\end{array}$ & $\begin{array}{l}17.0 \% \\
69.7 \%\end{array}$ & $(69.0 \pm 0.5) \%$ & $35.4 \%$ & $42.5 \%$ & $11.1 \%$ \\
\hline $\begin{aligned} & \text { Rest: } Q_{S V}<--0.8 \\
& l_{o p p}<2 \mathrm{~mm} \\
& l_{o p p}>2 \mathrm{~mm} \\
&\end{aligned}$ & $\begin{array}{l}96578 \\
25662\end{array}$ & $\begin{array}{r}19.5 \% \\
72.9 \% \\
\end{array}$ & $(72.5 \pm 0.8) \%$ & $38.5 \%$ & $40.5 \%$ & $10.5 \%$ \\
\hline
\end{tabular}

Table 1: Numbers of secondary vertices in the data and the sample compositions as predicted by the Monte Carlo. In total, six subsamples have been distinguished. The average values of the $\mathrm{b}$ quark and $\mathrm{b}$ hadron purities are shown, integrated over the entire impact parameter fit region. For the case of $l_{\text {opp }}>2 \mathrm{~mm}$, the predicted Monte Carlo b quark purities (first number) are compared with the fitted ones (second number). The values of the $\mathrm{B}^{+}, \mathrm{B}_{\mathrm{d}}^{0}$ and $\mathrm{B}_{\mathrm{s}}^{0}$ purities are normalised to the $\mathrm{b}$ quark content in each sample. 


\begin{tabular}{||l|r|r|r|r||}
\hline Error source & Error on $\Delta \Gamma / \Gamma$ & $\Delta \tau\left(\mathrm{B}^{+}\right)(\mathrm{fs})$ & $\Delta \tau\left(\mathrm{B}_{\mathrm{d}}^{0}\right)(\mathrm{fs})$ & $\Delta r$ \\
\hline \hline Detector Uncertainties & & & & \\
\hline Tracking efficiency & 0.009 & 2 & 5 & 0.005 \\
\hline Calibration and alignment & 0.012 & 5 & 11 & 0.011 \\
\hline Resolution parameter & 0.087 & 3 & 7 & 0.003 \\
\hline Resolution model & 0.095 & 11 & 15 & 0.005 \\
\hline Beam spot size and position & 0.010 & 3 & 10 & 0.009 \\
\hline Jet direction resolution & 0.052 & 5 & 7 & 0.002 \\
\hline \hline Total & 0.140 & 14 & 24 & 0.016 \\
\hline \hline Physics Model Uncertainties & & & & \\
\hline$R_{b}$ & 0.027 & 3 & 6 & 0.003 \\
\hline$R_{c}$ & 0.010 & 2 & 3 & 0.001 \\
\hline b fragmentation & 0.025 & 5 & 7 & 0.002 \\
\hline c fragmentation & 0.014 & 1 & 3 & 0.003 \\
\hline Transverse jet shape & 0.055 & 10 & 6 & 0.010 \\
\hline b decay multiplicity & 0.028 & 1 & 8 & 0.006 \\
\hline D fraction in cc events & 0.053 & 2 & 5 & 0.005 \\
\hline D content in b decays & 0.108 & 8 & 18 & 0.010 \\
\hline B $\rightarrow$ D D X rate & 0.021 & 15 & 11 & 0.002 \\
\hline D lifetimes & 0.018 & 2 & 4 & 0.002 \\
\hline B $/ B^{0}$ separation & 0.046 & 6 & 10 & 0.010 \\
\hline \hline Total & 0.151 & 22 & 28 & 0.020 \\
\hline \hline Grand Total & 0.206 & 26 & 37 & 0.026 \\
\hline \hline
\end{tabular}

Table 2: Systematic errors in the measurement of $\Delta \Gamma / \Gamma, \tau\left(\mathrm{B}^{+}\right), \tau\left(\mathrm{B}_{\mathrm{d}}^{0}\right)$ and the ratio $r=$ $\tau\left(\mathrm{B}^{+}\right) / \tau\left(\mathrm{B}_{\mathrm{d}}^{0}\right)$.

\begin{tabular}{|c|c|c|c|c|c|}
\hline Fit variable & Range & $\Delta \Gamma / \Gamma$ & $\begin{array}{l}\text { Contribut } \\
\text { statistica } \\
\tau\left(\mathrm{B}^{+}\right)(\mathrm{fs})\end{array}$ & $\begin{array}{l}\text { on to the } \\
\text { error of } \\
\tau\left(\mathrm{B}_{\mathrm{d}}^{0}\right)(\mathrm{fs})\end{array}$ & $r$ \\
\hline$\overline{\tau\left(\mathrm{B}_{\mathrm{S}}^{0}\right)}$ & $(1.49 \pm 0.06) \mathrm{fs}$ & 0.037 & 2 & 9 & 0.005 \\
\hline$\tau\left(\Lambda_{\mathrm{b}}\right)$ & $(1.22 \pm 0.05) \mathrm{fs}$ & 0.018 & 1 & 4 & 0.004 \\
\hline$f_{\mathrm{B}_{\mathrm{s}}^{0}}$ & $0.105_{-0.017}^{+0.018}$ & 0.049 & 5 & 1 & 0.004 \\
\hline$f_{\Lambda_{\mathrm{b}}}$ & $0.101_{-0.031}^{+0.039}$ & 0.037 & $\begin{array}{l}+11 \\
-8\end{array}$ & $\begin{array}{l}+16 \\
-12\end{array}$ & $\begin{array}{l}-0.004 \\
+0.003\end{array}$ \\
\hline$\left\langle n_{K}\right\rangle$ & $1.27 \pm 0.03$ & 0.057 & 1 & 4 & 0.002 \\
\hline
\end{tabular}

Table 3: Contributions to the statistical errors of $\Delta \Gamma / \Gamma, \tau\left(\mathrm{B}^{+}\right), \tau\left(\mathrm{B}_{\mathrm{d}}^{0}\right)$ and $r=\tau\left(\mathrm{B}^{+}\right) / \tau\left(\mathrm{B}_{\mathrm{d}}^{0}\right)$ from the uncertainties in the lifetimes $\tau\left(\mathrm{B}_{\mathrm{s}}^{0}\right), \tau\left(\Lambda_{\mathrm{b}}\right)$ and production fractions $f_{\mathrm{B}_{\mathrm{s}}^{0}}, f_{\Lambda_{\mathrm{b}}}$ of $\mathrm{B}_{\mathrm{s}}^{0}$ and $\Lambda_{\mathrm{b}}$ hadrons and the average rate $\left\langle n_{K}\right\rangle$ of $\mathrm{K}_{\mathrm{S}}^{0}$ and $\Lambda$ decays. 


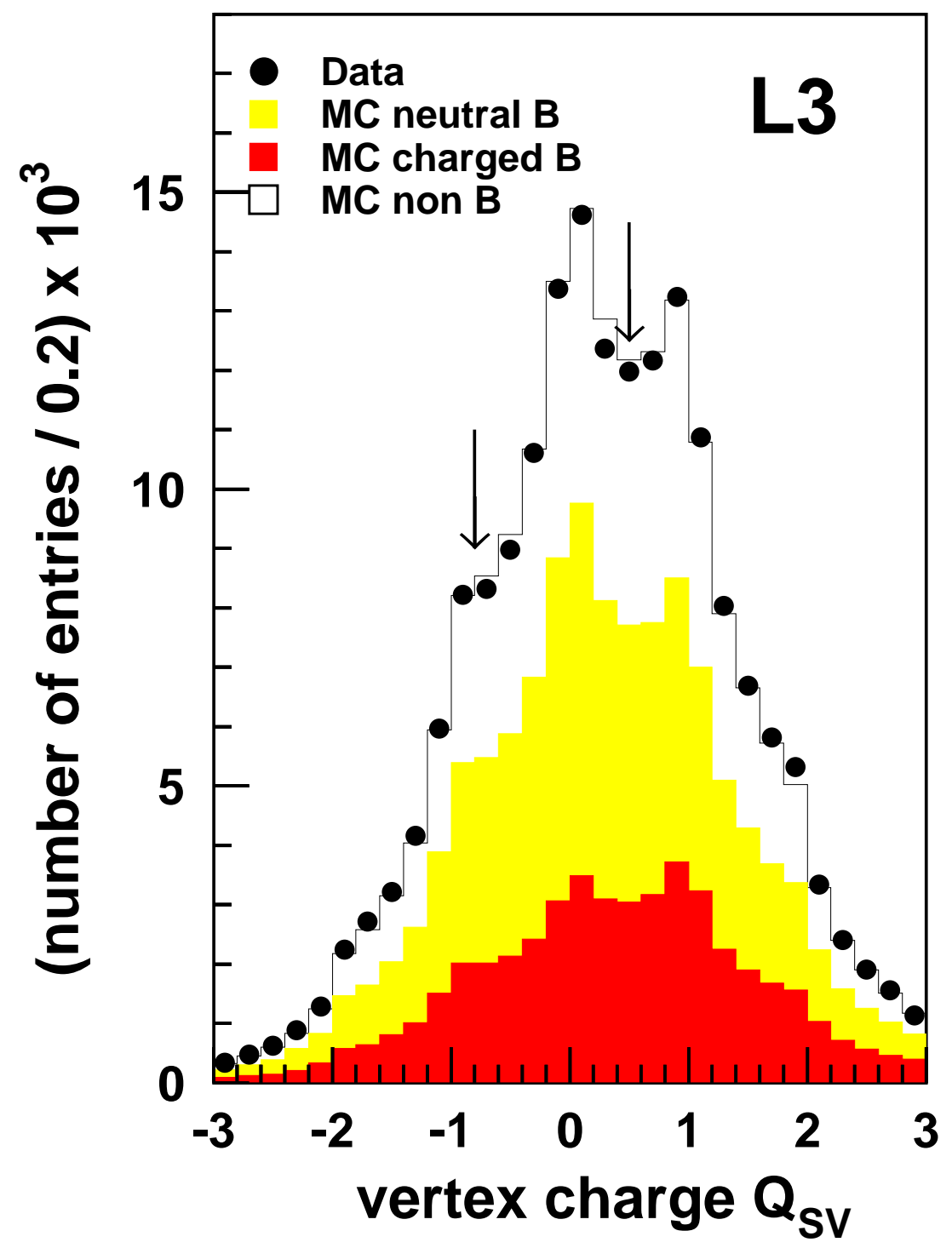

Figure 1: Secondary vertex charge distributions in the data (points) and Monte Carlo (histogram). The Monte Carlo contributions of neutral and charged b hadrons are shown separately. For this plot an enrichment of $b$ events has been obtained by a cut on the decay distance $l_{\text {opp }}$ in the opposite event hemisphere, $l_{\text {opp }}>2 \mathrm{~mm}$. The arrows indicate the positions of the cuts used to separate neutral $\left(-0.8<Q_{S V}<0.5\right)$ and charged $\left(Q_{S V}>0.5\right)$ samples. 


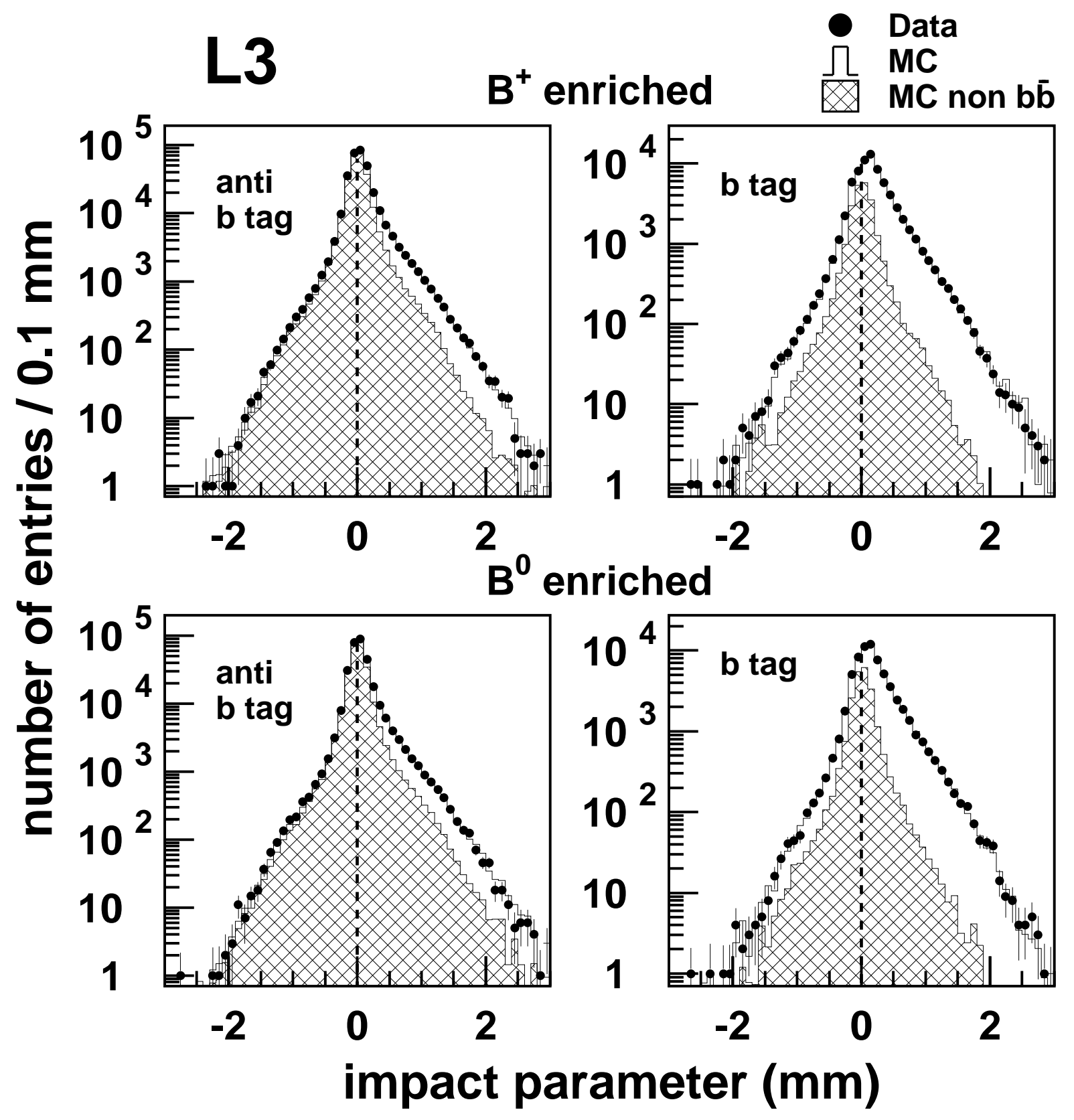

Figure 2: Impact parameter $\delta^{r \phi}$ distributions for four subsamples of the data. The upper plots show the distributions for the charged sample, the lower for the neutral sample in, respectively, a b-enriched sample (right) and a b-depleted sample (left). 


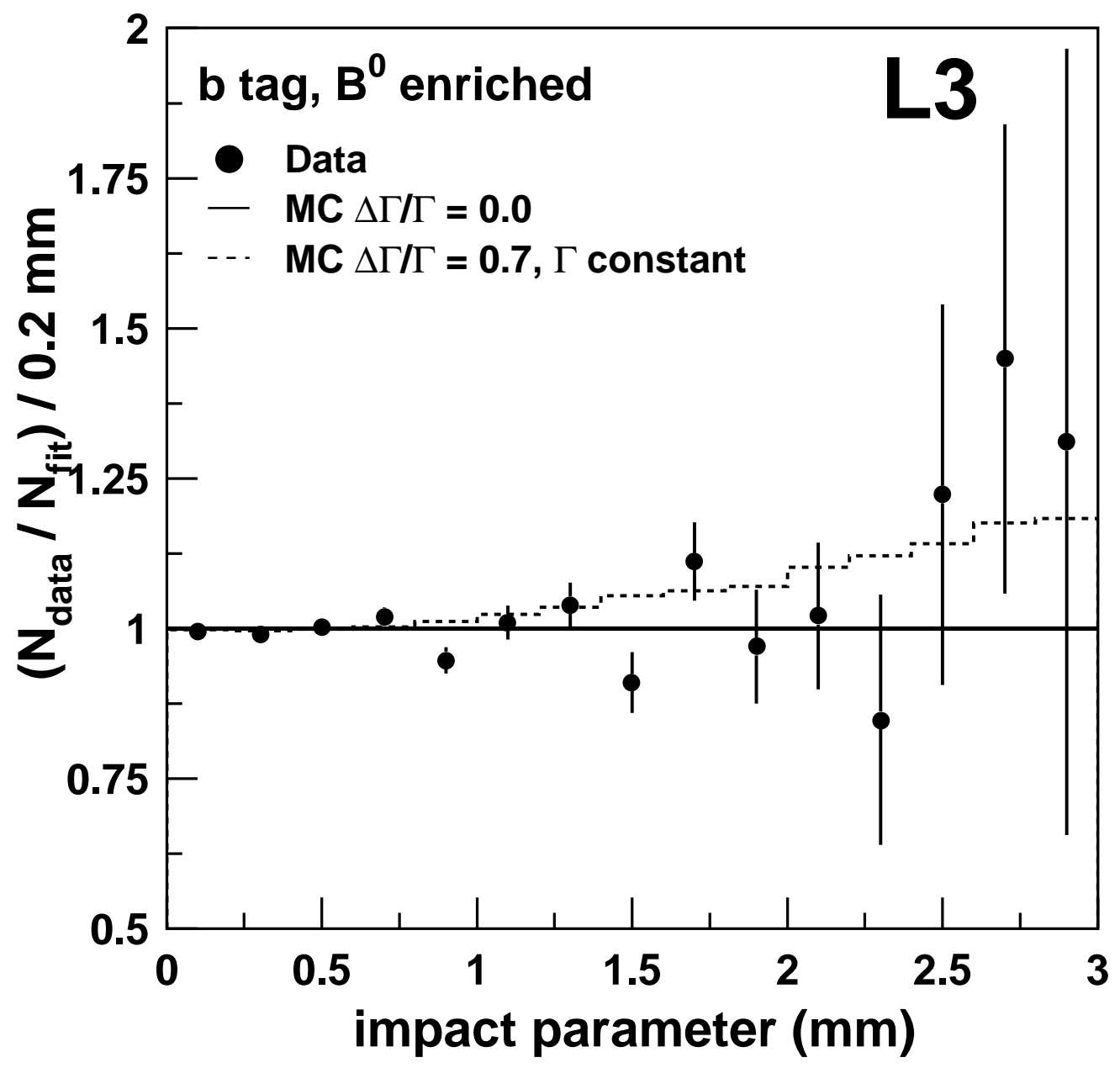

Figure 3: Dependence of the impact parameter fit on the rate difference $\Delta \Gamma / \Gamma$ of $B_{\mathrm{s}}^{0}$ mesons. The data distribution in the b-enriched neutral subsample (points) is normalised to the Monte Carlo distribution for the fitted $\Delta \Gamma / \Gamma$ value of $\Delta \Gamma / \Gamma=0$. The dashed line shows the expected Monte Carlo distribution for a value of $\Delta \Gamma / \Gamma=0.7$ where $\Gamma$ is fixed to the fitted value. 